OPEN ACCESS

Edited by:

Dusko Kozic,

University of Novi Sad, Serbia

Reviewed by:

Yann Quidé,

University of New South

Wales, Australia

Veena A. Nair,

University of Wisconsin-Madison,

United States

Ottavia Dipasquale,

King's College London,

United Kingdom

*Correspondence:

Joanna Bladowska

asia.bladowska@gmail.com;

joanna.bladowska@umed.wroc.pl

Specialty section

This article was submitted to Applied Neuroimaging,

a section of the journal

Frontiers in Neurology

Received: 24 December 2020

Accepted: 25 May 2021

Published: 12 July 2021

Citation:

Podgórski P, Waliszewska-Prosół M,

Zimny A, Sąsiadek M and Bladowska J (2021) Resting-State Functional Connectivity of the Ageing Female Brain-Differences Between Young and Elderly Female Adults on Multislice Short TR rs-fMRI

Front. Neurol. 12:645974. doi: 10.3389/fneur.2021.645974

\section{Resting-State Functional} Connectivity of the Ageing Female Brain-Differences Between Young and Elderly Female Adults on Multislice Short TR rs-fMRI

\author{
Przemysław Podgórski ${ }^{1}$, Marta Waliszewska-Prosół ${ }^{2}$, Anna Zimny ${ }^{1}$, Marek Sąsiadek ${ }^{1}$ and \\ Joanna Bladowska ${ }^{1 *}$
}

${ }^{1}$ Department of General and Interventional Radiology and Neuroradiology, Wroclaw Medical University, Wroclaw, Poland, ${ }^{2}$ Department of Neurology, Wroclaw Medical University, Wrocław, Poland

Introduction: Age-related brain changes are one of the most important world health problems due to the rising lifespan and size of the elderly populations. The aim of the study was to assess the effect of ageing in women on coordinated brain activity between eight resting-state networks.

Material and Methods: The study group comprised 60 healthy female volunteers who were divided into two age groups: younger women (aged 20-30 $n=30$ ) and older women (aged 55-80 $n=30$ ). Resting-state data were collected during a 15 min scan in the eyes-closed condition using a 3T MR scanner. Data were preprocessed and analysed using the CONN toolbox version 19.c. The large-scale network analysis included a priori selected regions of interest of the default mode, the sensorimotor, the visual, the salience, the dorsal attention, the fronto-parietal, the language, and the cerebellar network.

Results: Within the visual, the default mode, the salience, and the sensorimotor network, the intra-network resting-state functional connectivity (RSFC) was significantly higher with increasing age. There was also a significant increase in the inter-network RSFC in older females compared to young females found in the following networks: sensorimotor lateral and salience, salience and language, salience and fronto-parietal, cerebellar anterior and default mode, cerebellar posterior and default mode, visual and sensorimotor lateral, visual and sensorimotor, visual lateral and default mode, language and cerebellar anterior, language and cerebellar posterior, fronto-parietal and cerebellar anterior, dorsal attention and sensorimotor, dorsal attention and default mode, sensorimotor superior, and salience. Compared to young females, elderly women presented bilaterally significantly lower inter-network RSFC of the salience supramarginal gyrus and cerebellar posterior, sensorimotor lateral, and cerebellar anterior network, and sensorimotor lateral and cerebellar posterior as well as sensorimotor superior and cerebellar posterior network.

Conclusion: Increased RSFC between some brain networks including the visual, the default mode, the salience, the sensorimotor, the language, the fronto-parietal, the dorsal 
attention, and the cerebellar networks in elderly females may function as a compensation mechanism during the ageing process of the brain. To the best of our knowledge, this study is the first to report the importance of increase of cerebellar networks RSFC during healthy female ageing.

Keywords: ageing, functional connectivity, resting-state fMRI, brain networks, SPC, NBS, FNC, elderly women

\section{INTRODUCTION}

Ageing is one of the most important problems of contemporary society due to the increasing lifespan and size of elderly populations. There are known differences in the ageing process between women and men. Currently and historically, women tend to live longer than men worldwide according to reliable demographic data. On the other hand, women have a higher prevalence of chronic, age-related degenerative diseases than men (1). Sex differences influence brain morphology and physiology during both development and ageing. Men have larger brains in comparison with women $(2,3)$. At the same time, metabolic brain activity is significantly lower in females $(4,5)$. Furthermore, neuroanatomical sex differences in the brain are region-specific $(2,3)$. Apart from the anatomical dissimilarities, recently there have also been reports on differences associated with sex in resting-state functional connectivity $(\operatorname{RSFC})(6,7)$.

On the other hand, men more often turn to unhealthy behaviours such as smoking, use of psychoactive substances, poor diet, and alcohol consumption which contribute to the increased risk of cardiovascular, and other chronic diseases and higher mortality in men (8). In order to exclude the influence of cerebrovascular disease on resting-state RSFC changes, female subjects presenting a normal-appearing brain in conventional magnetic resonance imaging (MRI) seem to be suitable candidates to be included in a study group appropriate for the analysis of the normal brain ageing process.

Therefore, this paper explores the intra- and inter-network functional connectivity (FC) differences between the young female and the older female brain based on resting-state functional MRI (rs-fMRI) in order to avoid any impact of sex on age-related RSFC changes.

Resting-state fMRI enables the assessment of synchronous activations between brain areas that are spatially separate which occur in the absence of a stimulus or task. This method is noninvasive and does not require patient cooperation in order to identify resting-state networks in the brain $(9,10)$.

In general, in fMRI studies investigating resting-state networks, greater attention has been paid to the default mode network (DMN). The DMN contains a range of different cortical areas constantly found to be working at rest, including the medial prefrontal cortex, the inferior parietal lobule, the hippocampus, and the posterior cingulate cortex/retrosplenial cortex/precuneus (a group of regions also called the posteromedial cortex) (10). Moreover, the DMN plays a very important role in research studies concerning the ageing process, mainly due to special areas included in this network, such as the posterior cingulate cortex and the hippocampus, both of which are involved fundamentally in the pathogenesis of Alzheimer's disease $(10,11)$.

Obviously, the DMN is the most frequently investigated largescale resting-state network in the studies of age-related RSFC changes; however, the results are often inconclusive indicating that further investigations are needed $(10,12-14)$. Moreover, many reports on brain ageing have demonstrated decreased RSFC not only in the DMN but also within other networks, such as the salience network and the motor network (10,12, 15-18).

The aim of the study was to investigate the positive and negative effects of ageing in female adults on the coordinated activity between large-scale resting-state networks (RSNs) that are important for high-level cognitive functions. The purpose of our research was also to assess the influence of healthy ageing on RSFC network connectivity in women as well as trying to find out which network could act as a possible compensatory mechanism.

We performed an ROI-to-ROI analysis focusing on eight RSNs, including the DMN, the salience network (SAL), the fronto-parietal network (FPN), the dorsal attention network (DAN), the sensorimotor network (SMN), the language network (LAN), the visual network (VIS), and the cerebellar (CER) network.

\section{MATERIAL}

Sixty healthy female volunteers without neurological and cognitive deficits were enrolled in the study. The subjects were divided into two age groups: group 1-younger adults (aged 20-30 years; mean age 23.9 years; $n=30$ ) and group 2older adults (aged 55-80; mean age 63.3 years; $n=30$ ). The main radiological inclusion criterium was a normal-appearing brain on conventional MRI, with up to only five white matter hyperintensities.

Healthy right-handed female volunteers without past or present neurological, and cognitive deficits with at least +12 years of education and native Polish language skills. All participants had to be able to perform all activities of daily living independently.

All subjects in the study group had higher education and worked in their professions. Forty-five women (75\%) live in a large city (over 500,000 inhabitants), and the remaining 15 women (25\%) live in smaller towns and villages.

Additional demographic data are shown in Table 1.

The exclusion criteria were as follows:

1. Presence of any neurological disease (e.g., cerebrovascular disease, post-toxic changes, inflammatory changes, multiple sclerosis, brain neoplasms); 
TABLE 1 | Demographic and clinical data for both groups.

\begin{tabular}{lccc}
\hline & & & Statistics $\boldsymbol{t}$-test \\
\cline { 3 - 4 } Demographic data & Young women & Elderly women & $\boldsymbol{p}$ value \\
\hline Age & $23.9 \pm 2.9$ & $63.3 \pm 8.5$ & $<0.0001$ \\
Education (years) & $13.6 \pm 1.7$ & $14 \pm 1.9$ & 0.409 \\
EHI score & $78.5 \pm 21.2$ & $74.5 \pm 24.5$ & 0.508 \\
MMSE & $29.2 \pm 0.67$ & $28.8 \pm 0.76$ & 0.056 \\
CDT & 10 & 10 & - \\
\hline
\end{tabular}

EHI, Edinburgh Handedness Inventory; MMSE, Mini-Mental State Examination; CDT, Clock-drawing test.

2. Any autoimmune diseases, endocrinopathy and metabolic diseases (e.g., diabetes, cardiovascular diseases, systemic lupus erythematosus, Hashimoto's thyroiditis, Sjögren's syndrome);

3. A history of psychiatric disease (e.g., depression, schizophrenia, ADHD, autism);

4. Chronic use of CNS-active medications (e.g., neuroleptics, antiepileptic drugs, psychostimulants, steroids, analgesics, sedatives);

5. Active alcohol or drug abuse;

6. Visual and hearing impairment and/or symptoms of a neurological or cognitive deficit;

7. Any structural brain abnormality presented on conventional MR imaging;

8. Current pregnancy and breastfeeding, hormonal contraceptives;

9. A history of head trauma with loss of consciousness;

10. Being underweight $\left(\mathrm{BMI}<18.5 \mathrm{~kg} / \mathrm{m}^{2}\right)$ or obese with BMI $\geq 35 \mathrm{~kg} / \mathrm{m}^{2}$;

11. Claustrophobia.

\section{METHODS}

\section{Participants}

A prospective observational study was supported by the Wroclaw Medical University Grant SUB.C270.21.020 and conducted in accordance with the guidelines of the Wroclaw Medical University Ethics Committee for conducting research involving humans permission no. KB-57/2021. Each participant signed her informed consent before inclusion.

\section{Neurological Assessment}

The neurological study protocol included a detailed neurological examination, with assessment of mental state using the MiniMental State Examination (MMSE), and the clock-drawing test (CDT) to screen for cognitive impairment.

\section{Data Acquisition}

Resting-state data were collected during a 15 -min scan in a $3 \mathrm{~T}$ MR scanner (Ingenia Philips, Best, Netherlands) equipped with $45-\mathrm{mT} / \mathrm{m}$ 200-T/m/s gradients and a 32-channel head coil with foam padding to minimise head motion and noise-cancelling headphones. The study included the acquisition of a highresolution sagittal T1-weighted sequence (number of slices = 257; repetition time $(\mathrm{TR})=11 \mathrm{~ms}$; echo time $(\mathrm{TE})=5 \mathrm{~ms}$; flip angle $=8^{\circ}$; field of view $(\mathrm{FOV})=256 \times 256 \mathrm{~mm}$; and voxel size $=0.75 \times 0.75 \times 0.75 \mathrm{~mm})$. EPI multiband sequence: $\mathrm{MB}$ $=6, \mathrm{TR} / \mathrm{TE}=1,100 / 31 \mathrm{~ms}$, resolution $2.5 \times 2.5 \times 2.5 \mathrm{~mm}^{3}$ for rs-fMRI was performed. Participants were asked to stay awake during the examination with their eyes closed through the entire acquisition. After the rs-fMRI session, a verbal confirmation was used to cheque if they were awake during the rs-fMRI scanning procedure.

\section{Data Preprocessing}

Functional and structural images were analysed using CONN, a Matlab/SPM-based software. Data were preprocessed using a standard pipeline in Statistical Parametric Mapping software (SPM12, Wellcome Department of Cognitive Neurology, University College London) running under Matlab Release 2019b (The MathWorks, Inc., Natick, MA, United States) and Linux HPC Server running on Ubuntu 18.04. The preprocessing methods applied included slice timing correction, realignment, segmentation, normalisation to the MNI template, and smoothing. Functional data were realigned using SPM12 (19) and realign and unwarp procedure (20). All scans were co-registered and resampled to a reference image using b-spline interpolation. Temporal misalignment between different slices of the functional data was corrected using the SPM12 slice-timing correction procedure (21). Identification of outlier scans was performed using the Artefact Detection Tools (ART) toolbox (https://www.nitrc.org/projects/artifact_detect/). Outlier scans were identified from the observed global BOLD signal and the amount of subject motion in the scanner. Outlier scans that exceeded three standard deviations from the global mean BOLD signal, or with framewise displacement $>0.5 \mathrm{~mm}$, were identified. In the next step, functional and anatomical scans were normalised into standard MNI space with a final isotropic voxel size of $1 \mathrm{~mm}$ and segmented into grey matter, white matter, and cerebrospinal fluid tissue classes using SPM12 unified segmentation and normalisation procedure (22). The direct normalisation procedure applied unified segmentation and normalisation procedures separately to the functional data, using the mean BOLD signal as a reference image, and to the structural data, using the raw T1-weighted volume as a reference image. Both functional and anatomical data were resampled to a default $180 \times 216 \times 180$ - $\mathrm{mm}$ bounding box, with $2 \mathrm{~mm}$ isotropic voxels for functional data and $1 \mathrm{~mm}$ for anatomical data, using fourth-order spline interpolation. In order to minimise the impact of motion and physiological noise factors, the CompCor (23) function was used to define and remove confounds in the blood-oxygen-level-dependent signal. In order to focus on slow-frequency fluctuations, fMRI data were bandpass filtered at $0.008-0.09 \mathrm{~Hz}$ using a discrete cosine transform to minimise border effects (24).

Signal contributions from cerebrospinal fluid, brain white matter, and micro-movements of the head (three translation and three rotation parameters plus their associated first-order derivatives) were identified and removed by multiple regression. 
In order to increase the BOLD signal-to-noise ratio and reduce the influence of residual variability in functional and gyral anatomy across subjects, functional data were smoothed using spatial convolution with a Gaussian kernel of 8-mm full width at half maximum (FWHM).

\section{First-Level Analysis}

Thirty-two ROI seeds, with predefined shape and locations derived from the HCP atlas (25) adjusted to each volume, were used to assess eight RSNs including DMN, SAL, FPN, DAN, SMN, LAN, VIS, and CER (Figure 1). Functional connectivity measures were computed between earlier predefined seed regions of rs-networks to identify patterns of ROI-to-ROI connectivity by computing bivariate Pearson's correlation measures between the extracted mean BOLD signal time courses of each pair of ROIs. The resulting coefficients were converted to normally distributed scores using Fisher's transformation to improve normality assumptions of the subsequent, second-level analyses.

\section{Second-Level Analysis}

To allow between-group comparisons, individual matrices were entered into a second-level general linear model. We compared FC between group 1 and group 2 scans using two-tailed paired $t$-tests. The results were reported only when surviving a false discovery rate (FDR)-corrected threshold of $p<0.05$ at the seed level.

To address the issue of variability of the results of FC analyses which are partially dependent on the selected method used to control family-wise errors and node characteristics (26), we performed three separate analyses described in the statistics section comprising well-established methods such as functional network connectivity (FNC), spatial pairwise clustering (SPC), and network-based statistics (NBS).

\section{Statistical Analysis}

Between-subjects [Young Adults (-1) vs. Old Adults (1)], contrasts were applied for the ROI analysis. All network nodes were used as both sources and targets for the ROI analysis. Our findings are based on SPC analysis with the parameters described below. Additionally, we present alternative results by applying various approaches to node clustering and thresholding, such as FNC and NBS.

\section{FNC}

FNC is one of the default second-level analysis methods for the CONN toolbox that is based on an approach first described by Jafri et al. (27) used for determining the temporal dependency among the components with very weak relationships which cannot be considered as one component in the independent component analysis (ICA). FNC analyses the entire set of connexions between all pairs of ROIs in terms of intra- and internetwork connectivity sets (27). A multivariate parametric general linear model analysis for all connexions included in each of these sets/clusters of connexions was performed. As a final step for the obtained F-statistic for each pair of networks, a $p<0.05$ was used together with an associated uncorrected cluster-level height and an FDR-corrected cluster-level threshold of $p<0.05$ (28) to control FNC family-wise error rates.

\section{SPC}

The SPC approach is based on a work by Zalesky et al. (29). It starts with the entire ROI-to-ROI matrix of T- or F-statistics estimated using a general linear model, forming a twodimensional statistical parametric map which is then thresholded using an a priori "height" threshold. In the next step, resulting non-overlapping clusters are characterised by mass which is compared to a distribution of expected cluster mass values under the null hypothesis (25). The results are summarised, for each individual cluster or group of connexions, by uncorrected cluster-level $p$-values, cluster-level FWE-corrected $p$-values, and cluster-level FDR-corrected $p$-values. In our analyses, the following SPC parameters were used: cluster threshold: $p<0.05$ cluster-level p-FDR corrected (SPC mass/intensity); connexion threshold: $p<0.01 p$ uncorrected.

\section{NBS}

NBS is a method similar to SPC that allows making inferences about entire networks of ROIs. The clusters in NBS are defined by a graph theoretical concept of connected components (30) instead of pairwise clustering like in SPC. NBS analysis starts with the entire ROI-to-ROI matrix of T- or F-statistics estimated using a general linear model, forming a two-dimensional statistical parametric map which is then thresholded using an a priori "height" threshold in which order of ROIs is not relevant. Those resulting suprathreshold connexions define the graph among all nodes. In the next step, the obtained graph is broken into components/networks which are defined as connected subgraphs (25). Then, each network is characterised by its network mass but using networks instead of clusters as in SPC (25). Results are summarised, for each individual network or group of connexions, by uncorrected network-level $p$-values, network-level FWEcorrected $p$-values, and network-level FDR-corrected $p$-values.

The values used as thresholds in our study were as follows: network threshold: $p<0.05$ network-level $\mathrm{p}$ FDR corrected (NBS mass/intensity) connexion threshold: $p<0.001 p$-uncorrected.

\section{RESULTS}

\section{Neurological Examination and Cognitive Function}

The neurological examination and the results of the MMSE and CDT tests were within normal limits in all subjects (Table 1).

\section{Intra- and Inter-connectivity Pattern Analysis of Large-Scale Networks}

The ROI-ROI FC analysis using the SPC method revealed a significant increase in the intra- and inter-network brain FC within 11 clusters (Table 2) and a significant decrease in the internetwork brain FC in two clusters (Table 3 ) in group 2 (elderly females) in comparison to group 1 (younger females).

Figure 2 illustrates FC in younger females measured between rs-networks, while Figure 3 shows FNC in older women. 


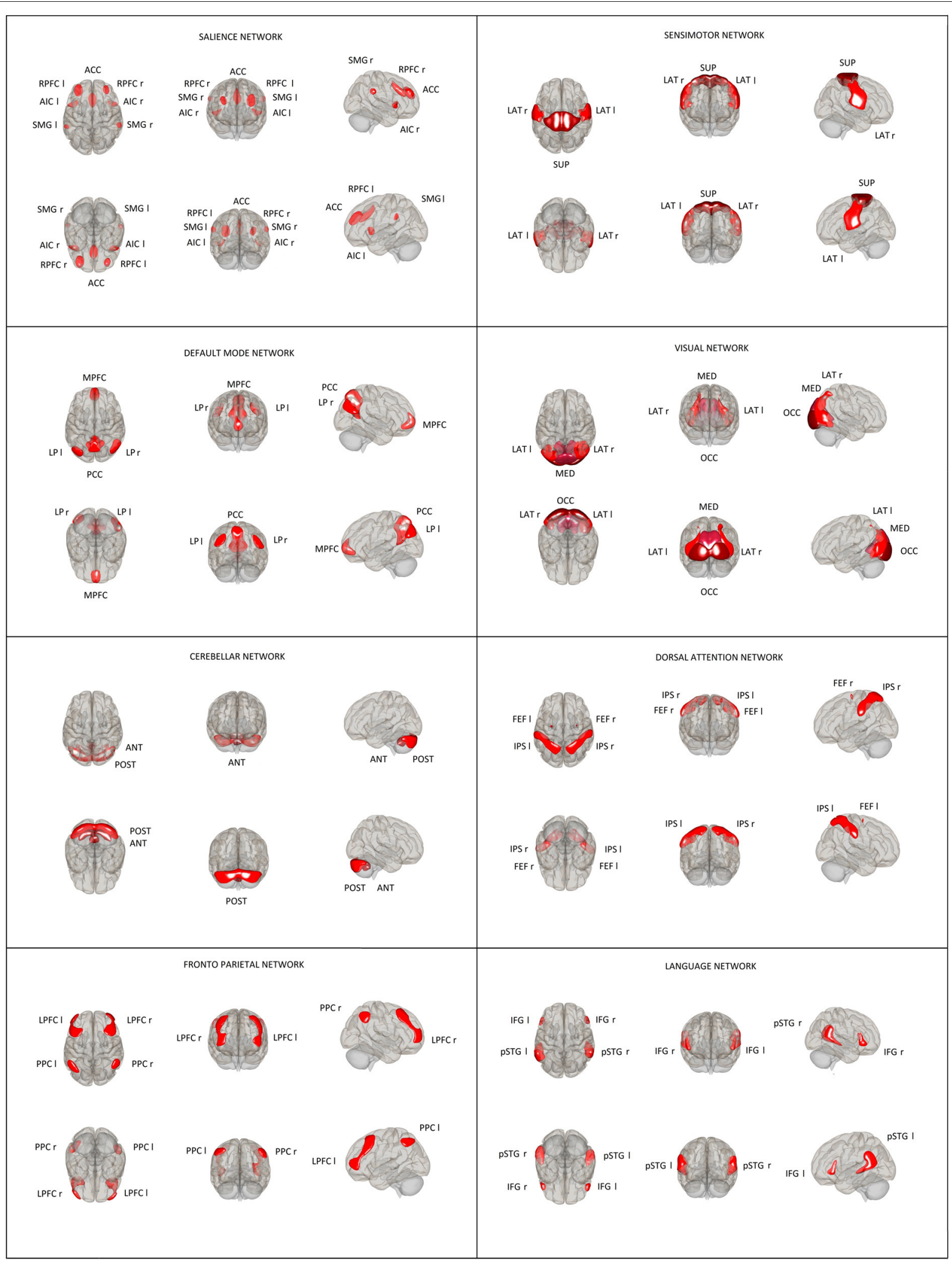

FIGURE 1 | Location and size of predefined rs-network ROls used in the ROI-ROI analysis. Default mode network (DMN): medial prefrontal cortex (MPFC), precuneus cortex (PCC), bilateral lateral parietal (LP); sensorimotor network (SMN): superior, bilateral lateral; visual network (VIS): medial, occipital, bilateral lateral; salience network (SN): anterior cingulate cortex (ACC), bilateral anterior insula (Al), rostral prefrontal cortex (RPFC), and supramarginal gyrus (SMG); dorsal attention network (DA): bilateral frontal eye field (FEF) and intraparietal sulcus (IPS); fronto-parietal network (FPN): bilateral lateral prefrontal cortex (LPFC) and posterior parietal cortex (PPC); language network (LAN): bilateral inferior frontal gyrus (IFG) and posterior superior temporal gyrus (pSTG); and cerebellar network (CER): anterior, posterior. 
TABLE 2 | Statistical cluster analysis of the networks using spatial pairwise clustering (SPC) presenting increased connectivity between nodes.

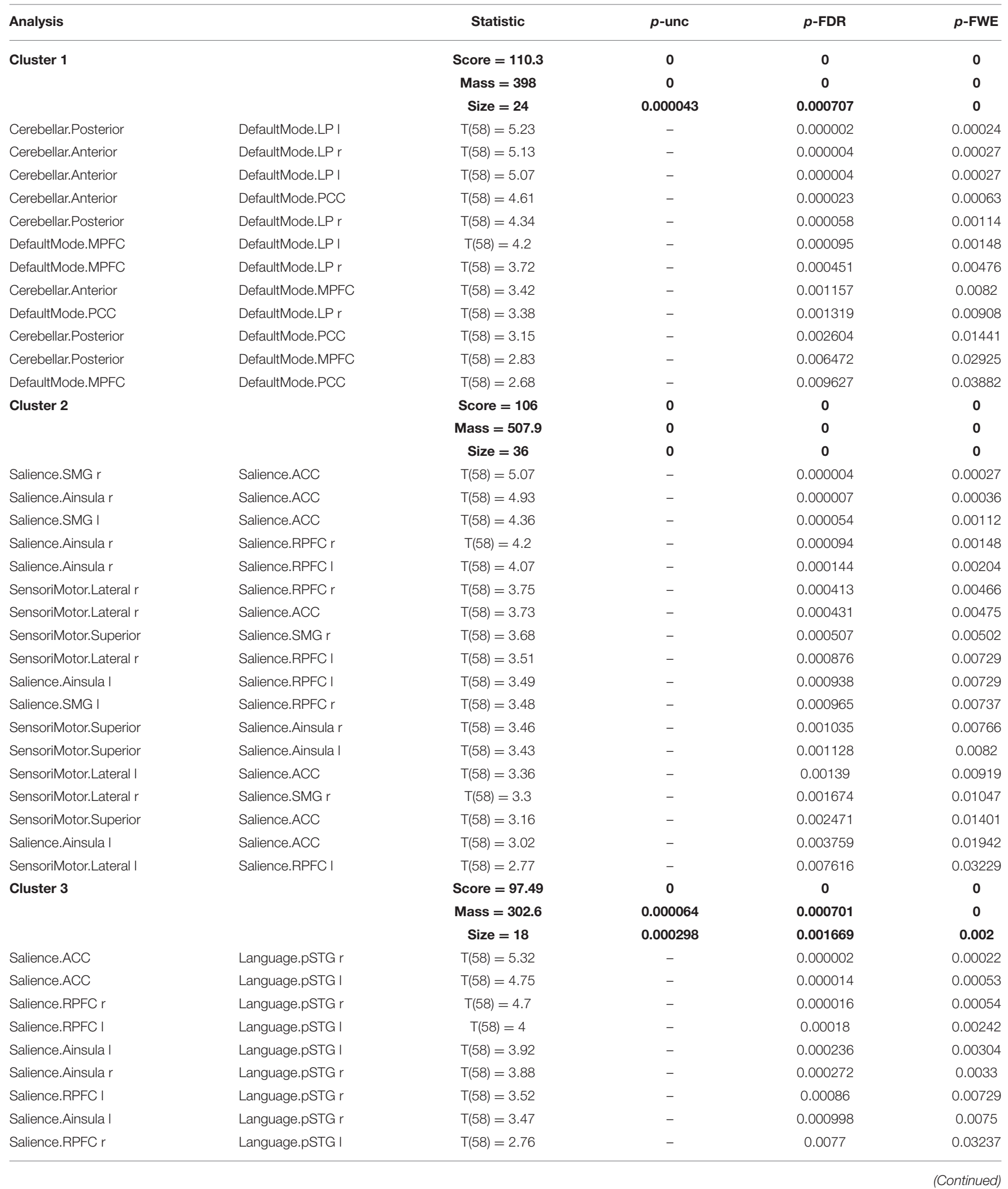


TABLE 2 | Continued

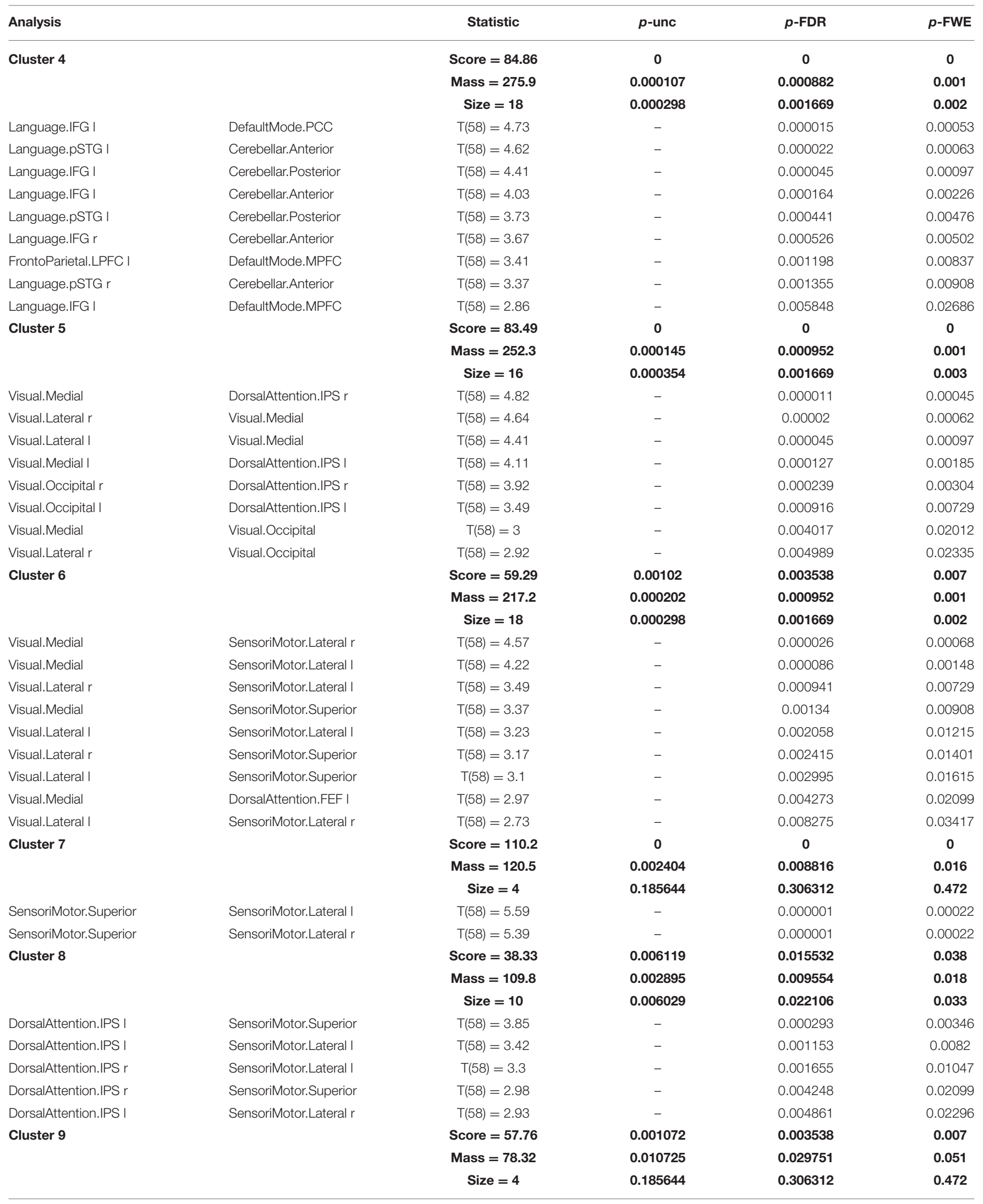


TABLE 2 | Continued

\begin{tabular}{|c|c|c|c|c|c|}
\hline Analysis & & Statistic & $p$-unc & $p$-FDR & $p$-FWE \\
\hline FrontoParietal.PPC I & Cerebellar.Anterior & $\mathrm{T}(58)=5.49$ & - & 0.000001 & 0.00022 \\
\hline FrontoParietal.PPC r & Cerebellar.Anterior & $\mathrm{T}(58)=3$ & - & 0.004004 & 0.02012 \\
\hline & & Mass $=78.02$ & 0.010818 & 0.029751 & 0.051 \\
\hline & & Size $=8$ & 0.013605 & 0.044896 & 0.064 \\
\hline DorsalAttention.FEF r & Salience.ACC & $\mathrm{T}(58)=2.88$ & - & 0.005612 & 0.02602 \\
\hline DorsalAttention.FEF I & Salience.RPFC I & $T(58)=2.69$ & - & 0.00941 & 0.03826 \\
\hline \multirow[t]{3}{*}{ Cluster 11} & & Score $=50.87$ & 0.001557 & 0.00467 & 0.01 \\
\hline & & Mass $=71.96$ & 0.013226 & 0.033574 & 0.062 \\
\hline & & Size $=4$ & 0.185644 & 0.306312 & 0.472 \\
\hline
\end{tabular}

$p$-unc, uncorrected $p$-value; $p$-FDR, false discovery rate-adjusted $p$-value.

$p$-FWE, family-wise error rate-adjusted $p$-value.

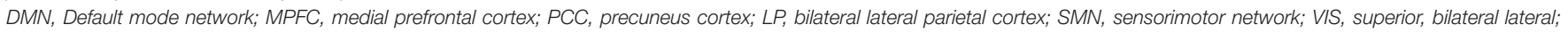

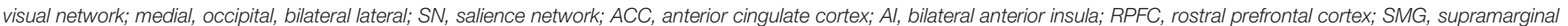

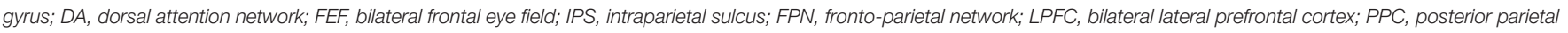
cortex; LAN, language network; IFG, bilateral inferior frontal gyrus; pSTG, posterior superior temporal gyrus; CER, cerebellar network: anterior, posterior.

The bold values are cluster statistic.

TABLE 3 | Statistical cluster analysis of the networks using spatial pairwise clustering (SPC) showing decreased connectivity between nodes.

\begin{tabular}{|c|c|c|c|c|c|}
\hline & & & $p$-unc & $p$-FDR & $p$-FWE \\
\hline \multirow[t]{2}{*}{ Cluster 1} & & 70.38 & 0.000474 & 0.002233 & 0.002 \\
\hline & & Mass $=225.1$ & 0.000189 & 0.000952 & 0.001 \\
\hline Salience.SMG r & Cerebellar.Posterior & $\mathrm{T}(58)=-4.95$ & - & 0.000007 & 0.00036 \\
\hline SensoriMotor.Lateral $r$ & Cerebellar.Anterior & $\mathrm{T}(58)=-4.14$ & - & 0.000113 & 0.00169 \\
\hline Salience.SMG I & Cerebellar.Posterior & $T(58)=-3.54$ & - & 0.000803 & 0.00699 \\
\hline SensoriMotor.Lateral I & Cerebellar.Posterior & $\mathrm{T}(58)=-3.31$ & - & 0.001629 & 0.01047 \\
\hline SensoriMotor.Superior & Cerebellar.Posterior & $\mathrm{T}(58)=-3.26$ & - & 0.001873 & 0.01147 \\
\hline SensoriMotor.Lateral r & Cerebellar.Posterior & $\mathrm{T}(58)=-3.23$ & - & 0.00205 & 0.01215 \\
\hline Cluster 2 & & Score $=62.79$ & 0.000691 & 0.002849 & 0.004 \\
\hline Salience.SMG r & Language.IFG I & $T(58)=-4.33$ & - & 0.00006 & 0.00114 \\
\hline Salience.SMG I & Language.IFG I & $\mathrm{T}(58)=-3.68$ & - & 0.000518 & 0.00502 \\
\hline Salience.SMG I & FrontoParietal.LPFC I & $T(58)=-3.66$ & - & 0.000554 & 0.00519 \\
\hline Salience.Ainsula $r$ & FrontoParietal.LPFC I & $\mathrm{T}(58)=-3.06$ & - & 0.003366 & 0.01772 \\
\hline Salience.SMG r & Language.pSTG I & $\mathrm{T}(58)=-3$ & - & 0.004016 & 0.02012 \\
\hline Salience.Ainsula $r$ & Language.IFG | & $\mathrm{T}(58)=-2.73$ & - & 0.008322 & 0.03417 \\
\hline
\end{tabular}

$p$-unc, uncorrected $p$-value; $p$-FDR, false discovery rate-adjusted $p$-value; $p$-FEW, family-wise error rate-adjusted $p$-value.

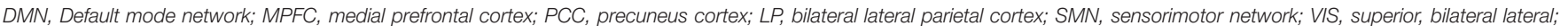

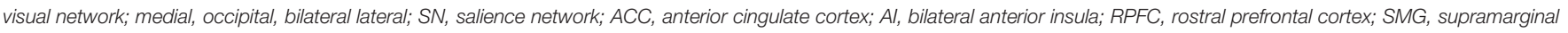

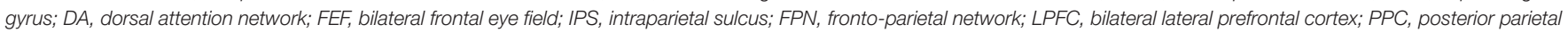
cortex; LAN, language network; IFG, bilateral inferior frontal gyrus; pSTG, posterior superior temporal gyrus; CER, cerebellar network: anterior, posterior.

The bold values are cluster statistic. 


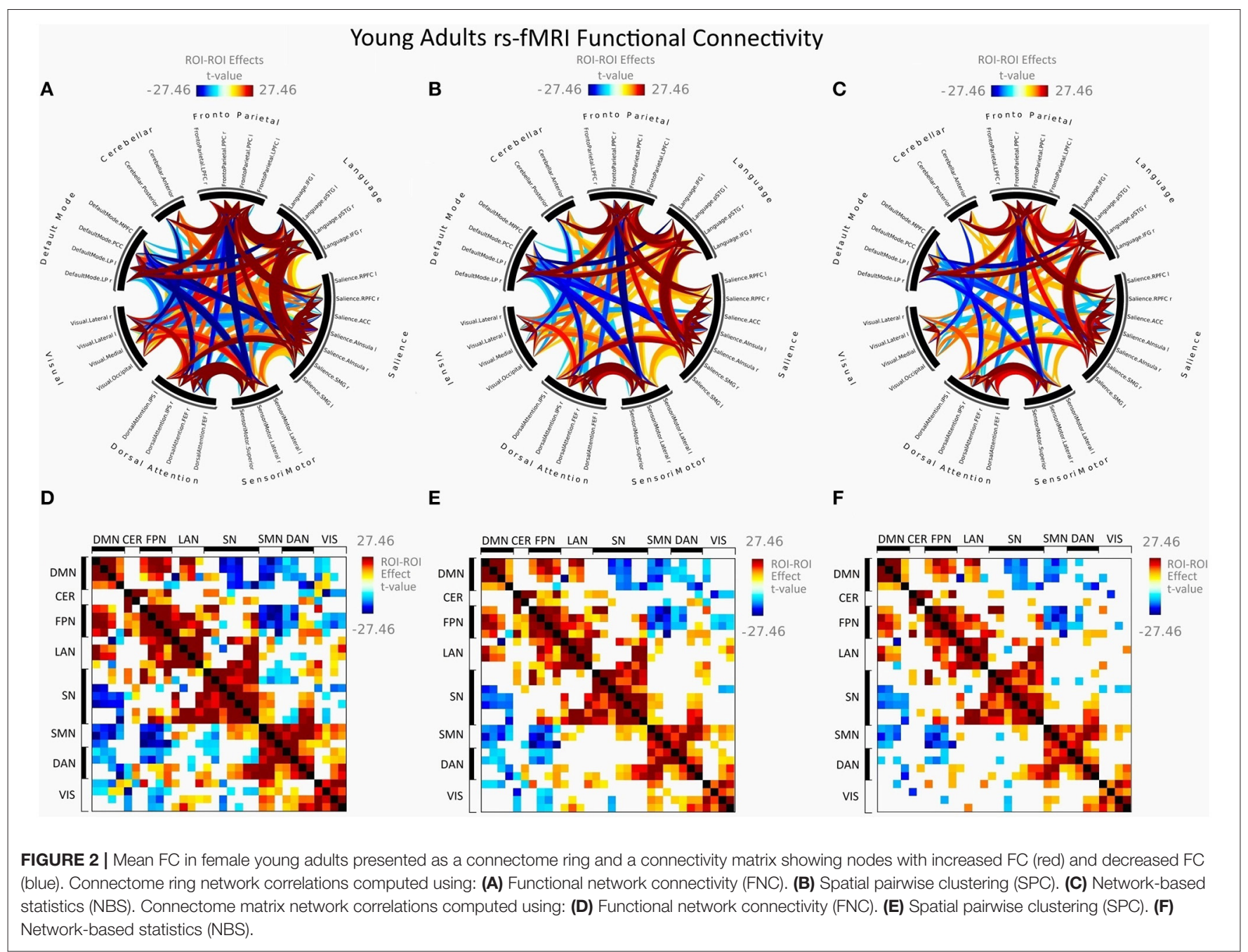

The differences between groups in FC including eight main rs-networks are presented in Figure 4.

\section{Increase in Intra-Network Brain Functional Connectivity Related to Older Age}

There was a significant increase in the intra-network brain FC in older females (group 2) compared to young females (group 1) found in the following nodes of networks: the default mode $(\mathrm{DMN})$, the salience $(\mathrm{SN})$, the sensorimotor $(\mathrm{SMN})$, and the visual (VIS) network.

Figure 5 shows increased intra-network connectivity in females with ageing.

\section{Increase in Inter-network Brain Functional Connectivity Related to Older Age}

Group 2 (elderly females) presented a significantly increased inter-network brain FC bilaterally of sensorimotor lateral and salience rostral prefrontal cortex (RPFC), salience and language, salience and fronto-parietal, cerebellar anterior and default mode, cerebellar posterior and default mode, visual and sensorimotor lateral, visual lateral and default mode, language and cerebellar anterior, language and cerebellar posterior, fronto-parietal and cerebellar anterior, dorsal attention and sensorimotor, dorsal attention and default mode, and sensorimotor superior and salience networks (Figure 6).

Apart from that, the second group revealed a significant increase in the inter-network brain FC only on one side between: language inferior frontal gyrus (IFG) and right fronto-parietal posterior parietal cortex (PPC), left language IFG and default mode precuneus cortex (PCC), left language IFG and default mode medial prefrontal cortex (MPFC), right dorsal attention intraparietal sulcus (IPS), and right salience RPFC.

The results concerning significant increase in the intra- and inter-network brain FC are shown in Table 2.

\section{Decrease in Inter-network Brain Functional Connectivity Related to Older Age}

Compared to group 1 (female young adults), group 2 (female older adults) presented a significantly decreased inter-network brain FC bilaterally of the salience supramarginal gyrus (SMG) 


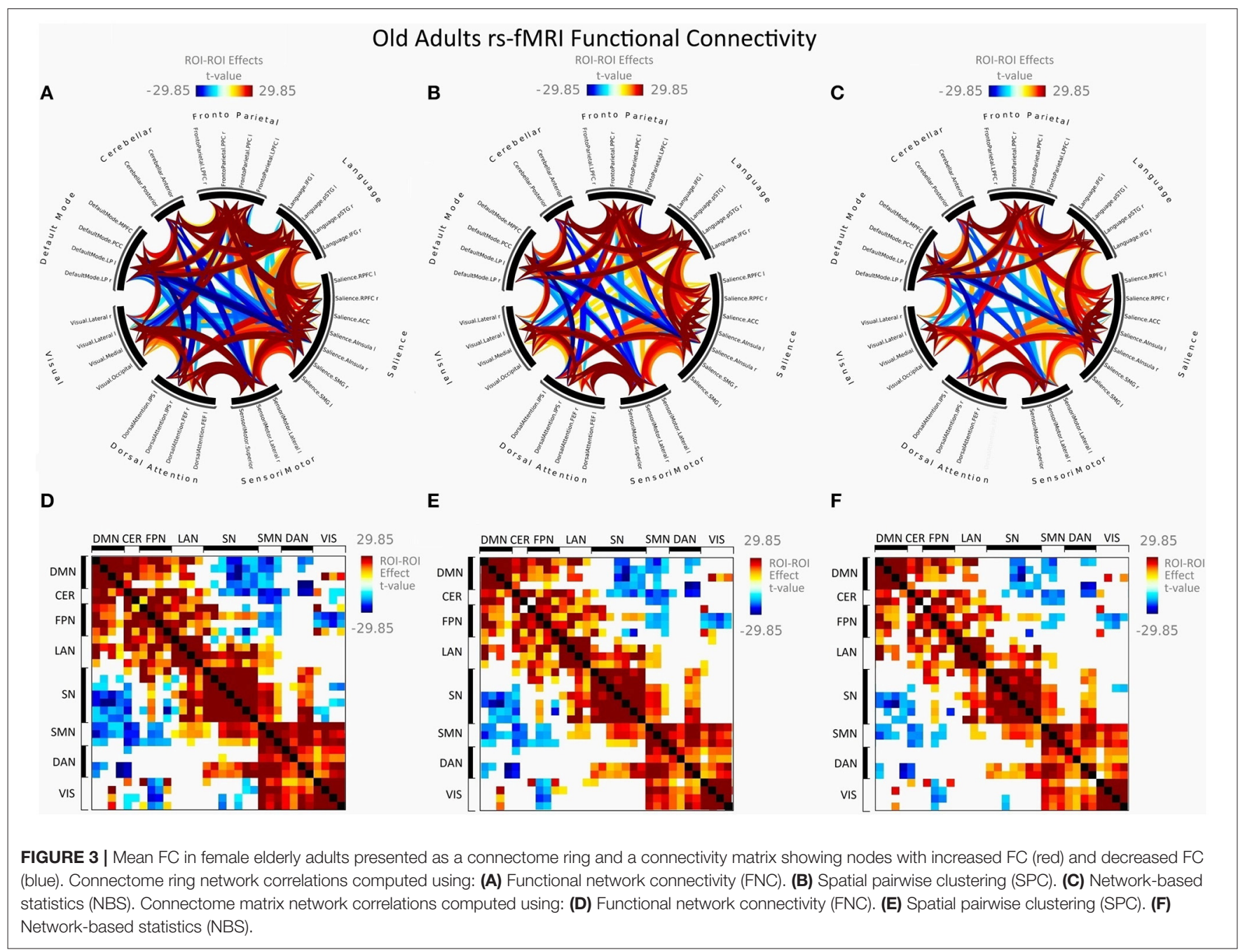

and the cerebellar posterior, the sensorimotor lateral and the cerebellar anterior networks, the sensorimotor lateral and the cerebellar posterior network, and the sensorimotor superior and cerebellar posterior network.

Additionally, group 2 showed a significant decrease in the inter-network brain FC only on one side between: dorsal attention IPS and cerebellar posterior, salience SMGlanguage IFG, right salience SMG and left fronto-parietal lateral prefrontal cortex (LPFC), and left salience SMG and left frontoparietal LPFC.

The results concerning significant decrease in the internetwork brain FC are shown in Table 3 as well as in Figure 6.

\section{Stabilisation in Inter-network Brain Functional Connectivity With Age}

We found that some regions retained their FC characteristics with age. Several nodes were not significantly interconnected and remained the same as in the younger age. Those regions include such connexions as bilateral lateral parietal cortexfronto-parietal posterior parietal cortex (PPC) bilateral as well as default mode bilateral lateral parietal cortex-salience rostral prefrontal cortex (RPFC) bilateral.

\section{Comparison of the Results Obtained by Applying Various Approaches to Node Clustering and Thresholding: SPC vs. FNC, SPC vs. NBS, and NBS vs. SPC}

Table 4 shows the significantly increased ROI-ROI FC in older women found in the SPC analysis in comparison to the FNC method in the networks: left fronto-parietal posterior parietal cortex (PPC) and cerebellar anterior as well as right frontoparietal posterior parietal cortex (PPC) and cerebellar anterior.

Additionally, older women presented a significant increase in FC obtained with the SPC method and not observed in the NBS analysis between the following networks: cerebellar and default mode, sensorimotor and salience, visual and sensorimotor, dorsal attention and sensorimotor, dorsal attention, and salience as well as in the intra-network FC of the default mode, salience and VIS. A detailed comparison is shown in Table 5.

The significantly decreased FC between networks in group 2 observed in the SPC analysis but not in NBS are as follows: 


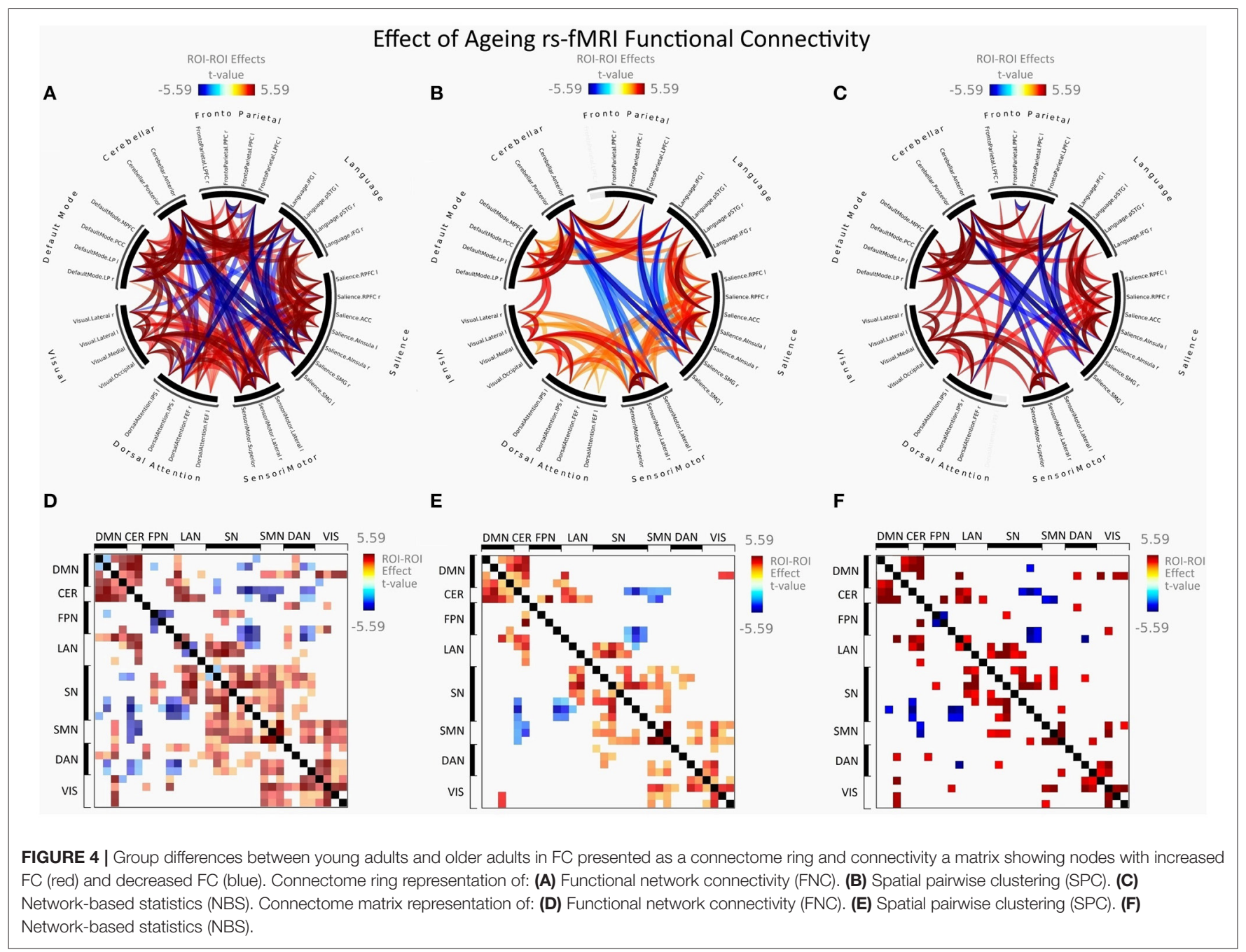

\section{Intra-Network Connectivity}

$\begin{aligned} & \text { Default Mode Network } \\ & \text { DefaultMode.MPFC }\end{aligned}$
$\begin{aligned} & \text { DefaultMode.LP I } \\ & \text { DefaultMode.LP r } \\ & \text { DefaultMode.PCC } \\ & \text { DefaultMode.LP r }\end{aligned}$

Increased connectivity between nodes
Decreased connectivity between node

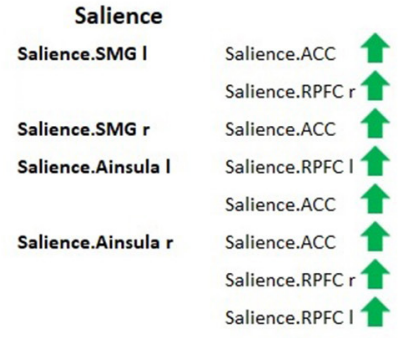

Sensimotor

SensoriMotor.Superior SensoriMotor.Lateral 1

SensoriMotor.Superior SensoriMotor.Lateral $r$ 1

\section{Visual Network}

Visual.Lateral $\mathbf{r}$ Visual.Media

Visual.Laterall Visual.Medial

Visual.Medial Visual.Occipital

Visual.Lateral r Visual.Occipital 1

FIGURE 5 | Intra-network connectivity table showing increased FC between nodes in females with ageing, using spatial pairwise clustering (SPC) method.

between salience and fronto-parietal as well as between salience and LAN (Table 6).

Older women showed a significantly increased inter-network FC with the NBS method, not found in the SPC analysis, between salience and fronto-parietal, dorsal attention and default mode, visual and fronto-parietal, and visual and default mode (Table 7).

Furthermore, the NBS analysis revealed a significantly decreased FC in older females between the dorsal attention and 


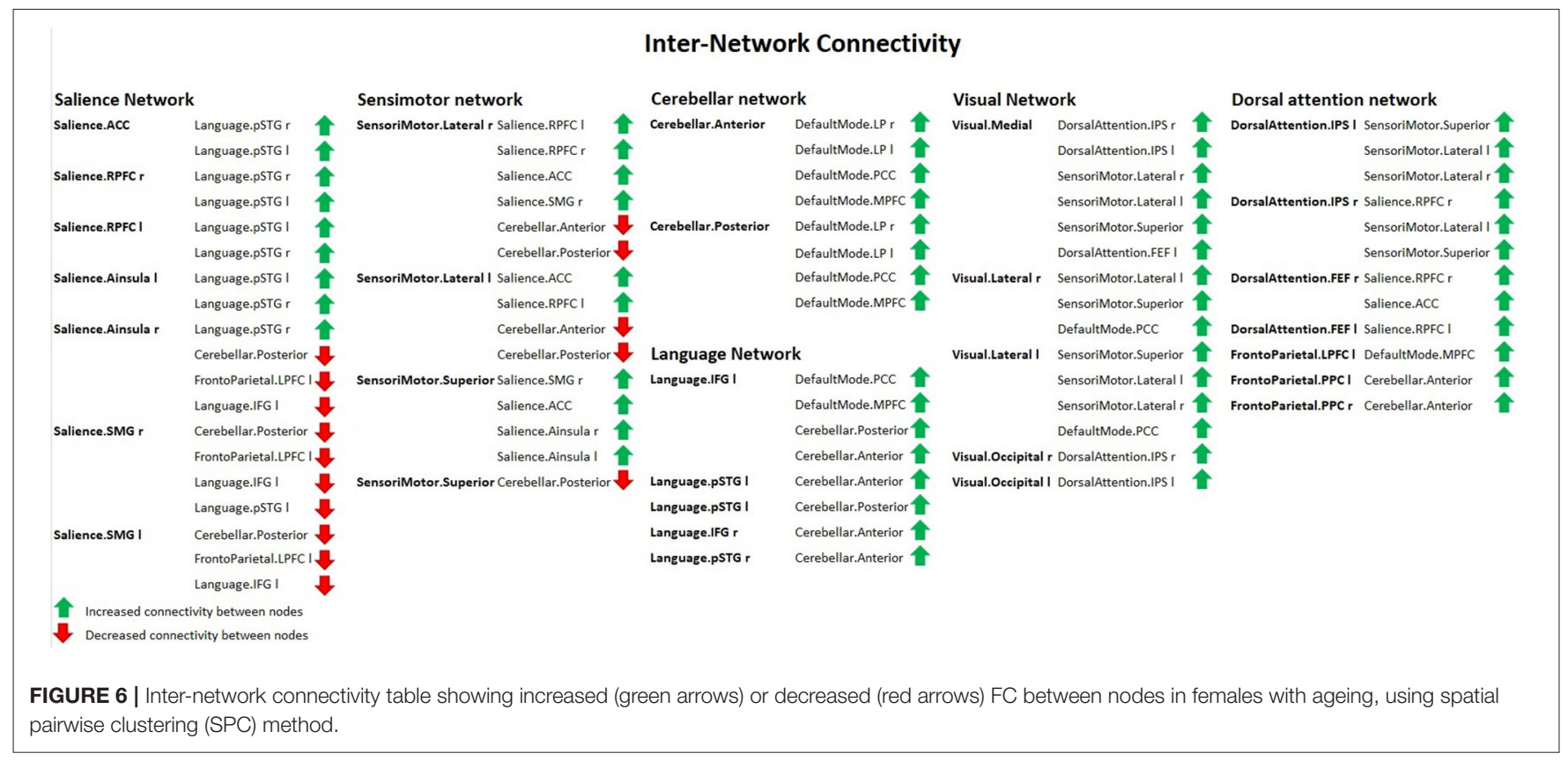

TABLE 4 | The increased functional connectivity in older females observed only in SPC analysis, not found in FNC method.

\begin{tabular}{llccc}
\hline & & \multicolumn{3}{c}{ SPC vs. FNC* } \\
\cline { 3 - 5 } & & T(58) & $\boldsymbol{p}$-FDR & $\boldsymbol{p}$-FWE \\
\hline FrontoParietal.PPC I & Cerebellar.Anterior & 5.49 & 0.000001 & 0.000219 \\
FrontoParietal.PPC r & Cerebellar.Anterior & 3 & 0.004004 & 0.020124
\end{tabular}

*Additional changes in connectivity observed in SPC and not presented as results in FNC analysis.

the LAN, the salience and the DMN as well as in the intranetwork FC of the FPN, as shown in Table 8.

In summary, the FNC method presented the highest number of results, the NBS approach was the most restrictive one, while SPC analysis showed intermediate results with the exceptions presented in Tables 5-8, which may be explained by different statistical approaches to cluster definition and family-wise error control.

Detailed results obtained with the FNC (Supplementary Table 1), and NBS method (Supplementary Table 2) are available as Supplementary Materials.

\section{DISCUSSION}

Overall, most of the studies which assessed the ageing process with the use of rs-MRI reported age-related impairment in RSFC with a special focus on the DMN (10, 12-14, 31-38). The authors concluded that, in general, ageing is connected with decrease within the DMN. However, there have been articles showing both increase and decrement in RSFC related to older age (39). On the other hand, one study did not reveal any effects of ageing on RSFC in the DMN (40), but this research should not be taken into account as the study group comprised only elderly subjects without comparison to younger adults.

\section{Increase in Intra- and Inter-Network Brain FC With Ageing}

In our study, we found a significant increase in the intra- and inter-network brain FC within the DMN in elderly females compared to the younger ones. We assume that the results could be associated with the method we used. Our analysis comprised networks of ROIs defined with the CONN network atlas. It should be stressed that the hippocampus region was not included within the detailed ROI assessment. Nevertheless, regarding the analysed networks, our results revealed that older women showed an increase compared with younger females in the intra- and inter-network brain FC within the DMN.

Apart from that, most of the reports published in the literature evaluated the age-related differences in RSFC without distinguishing between men and women. According to current science, little is known about sex differences in RSFC $(41,42)$. Zonneveld et al. (41) observed higher connectivity within the DMN as well as higher connectivity within the sensorimotor and VISs in females, which is in accordance with our findings.

Interestingly enough, ageing is accompanied by an increase in some of the processes that contribute to image processing in the brain. The integration of sensory information is optimised with ageing. Nerve networks in the brain restructure in response to the decrease of some sensorial functions $(43,44)$. Occipital activation by visual perception tends to decrease with age. In parallel to this, activation of the areas associated with 
TABLE 5 | The significant increase in functional connectivity in older females obtained only in SPC analysis, not found in NBS method.

\begin{tabular}{|c|c|c|c|c|}
\hline & & \multicolumn{3}{|c|}{ SPC vs. NBS* } \\
\hline & & $\mathrm{T}(58)$ & $p$-FDR & $p$-FWE \\
\hline Cerebellar.Anterior & DefaultMode.MPFC & 3.42 & 0.001157 & 0.003585 \\
\hline DefaultMode.PCC & DefaultMode.LP r & 3.38 & 0.001319 & 0.005839 \\
\hline Cerebellar.Posterior & DefaultMode.PCC & 3.15 & 0.002604 & 0.006727 \\
\hline Cerebellar.Posterior & DefaultMode.MPFC & 2.83 & 0.006472 & 0.013375 \\
\hline DefaultMode.MPFC & DefaultMode.PCC & 2.68 & 0.009627 & 0.042636 \\
\hline SensoriMotor.Superior & Salience.Ainsula r & 3.46 & 0.001035 & 0.005826 \\
\hline SensoriMotor.Superior & Salience.Ainsula I & 3.43 & 0.001128 & 0.005826 \\
\hline SensoriMotor.Lateral I & Salience.ACC & 3.36 & 0.00139 & 0.006415 \\
\hline SensoriMotor.Lateral r & Salience.SMG r & 3.3 & 0.001674 & 0.007412 \\
\hline SensoriMotor.Superior & Salience.ACC & 3.16 & 0.002471 & 0.007661 \\
\hline Salience.Ainsula I & Salience.ACC & 3.02 & 0.003759 & 0.023305 \\
\hline SensoriMotor.Lateral I & Salience.RPFC I & 2.77 & 0.007616 & 0.021464 \\
\hline Salience.RPFC r & Language.pSTG I & 2.76 & 0.0077 & 0.029838 \\
\hline FrontoParietal.LPFC I & DefaultMode.MPFC & 3.41 & 0.001198 & 0.009284 \\
\hline Language.pSTG r & Cerebellar.Anterior & 3.37 & 0.001355 & 0.007001 \\
\hline Language.IFG I & DefaultMode.MPFC & 2.86 & 0.005848 & 0.022661 \\
\hline Visual.Medial & Visual.Occipital & 3 & 0.004017 & 0.013006 \\
\hline Visual.Lateral r & Visual.Occipital & 2.92 & 0.004989 & 0.030932 \\
\hline Visual.Medial & SensoriMotor.Superior & 3.37 & 0.00134 & 0.005193 \\
\hline Visual.Lateral I & SensoriMotor.Lateral I & 3.23 & 0.002058 & 0.021263 \\
\hline Visual.Lateral r & SensoriMotor.Superior & 3.17 & 0.002415 & 0.018718 \\
\hline Visual.Lateral I & SensoriMotor.Superior & 3.1 & 0.002995 & 0.023211 \\
\hline Visual.Medial & DorsalAttention.FEF I & 2.97 & 0.004273 & 0.013006 \\
\hline Visual.Lateral I & SensoriMotor.Lateral r & 2.73 & 0.008275 & 0.051306 \\
\hline DorsalAttention.IPS I & SensoriMotor.Lateral I & 3.42 & 0.001153 & 0.008939 \\
\hline DorsalAttention.IPS r & SensoriMotor.Lateral I & 3.3 & 0.001655 & 0.010264 \\
\hline DorsalAttention.IPS I & SensoriMotor.Lateral r & 2.93 & 0.004861 & 0.025115 \\
\hline FrontoParietal.PPC r & Cerebellar.Anterior & 3 & 0.004004 & 0.020124 \\
\hline DorsalAttention.FEF r & Salience.RPFC r & 3.35 & 0.001412 & 0.014595 \\
\hline DorsalAttention.FEF r & Salience.ACC & 2.88 & 0.005612 & 0.043496 \\
\hline DorsalAttention.FEF I & Salience.RPFC I & 2.69 & 0.00941 & 0.05834 \\
\hline
\end{tabular}

*Additional changes in FC observed in SPC and not presented as results in NBS analysis.

TABLE 6 | The significant decrease in functional connectivity in older females observed only in SPC analysis, not revealed in NBS method.

\begin{tabular}{llccc}
\hline & & \multicolumn{3}{c}{ SPC vs. NBS* } \\
\cline { 3 - 5 } & & T(58) & p-FDR & p-FWE \\
\hline Salience.Ainsula r & FrontoParietal.LPFC I & -3.06 & 0.003366 & 0.011595 \\
Salience.SMG r & Language.pSTG I & -3 & 0.004016 & 0.015564 \\
Salience.Ainsula r & Language.IFG I & -2.73 & 0.008322 & 0.025797 \\
\hline
\end{tabular}

${ }^{*}$ Additional changes in FC observed in SPC and not present as results in NBS analysis.

control functions, particularly in the prefrontal cortex, increases (45). This reorganisation enables a compensatory recruitment of the structures that integrate the endogenous process of analysis (44).
TABLE 7 | The increased functional connectivity in older women observed only in NBS analysis, not found in SPC method.

\begin{tabular}{llccc}
\hline & & \multicolumn{3}{c}{ NBS vs. SPC* } \\
\cline { 3 - 5 } & & T(58) & $\boldsymbol{p}$-FDR & p-FWE \\
\hline Language.pSTG I & Language.IFG I & 3.56 & 0.000746 & 0.00673 \\
Salience.ACC & FrontoParietal.PPC $r$ & 3.49 & 0.000916 & 0.007294 \\
DorsalAttention.FEF r & DefaultMode.PCC & 3.8 & 0.000351 & 0.004045 \\
Visual.Medial & FrontoParietal.LPFC I & 3.88 & 0.000269 & 0.003295 \\
Visual.Occipital & DefaultMode.PCC & 4.53 & 0.00003 & 0.000735
\end{tabular}

${ }^{*}$ Additional changes in FC observed in NBS and not presented as results in SPC analysis.

TABLE 8 | The decreased functional connectivity in older women observed only in NBS analysis, not found in the SPC method.

\begin{tabular}{lllll}
\hline & & \multicolumn{3}{c}{ NBS vs. SPC* } \\
\cline { 3 - 5 } & & T(58) & p-FDR & p-FWE \\
\hline DorsalAttention.IPS r & Language.IFG I & -4.48 & 0.000035 & 0.000836 \\
FrontoParietal.PPC I & FrontoParietal.PPC r & -4.19 & 0.000095 & 0.001478 \\
Salience.SMG r & DefaultMode.LPI & -3.54 & 0.000785 & 0.006954
\end{tabular}

${ }^{*}$ Additional changes in FC observed in NBS and not presented as results in SPC analysis.

We found increased intra-network as well as inter-network brain FC related to older age within the VIS in women. Our results are consistent with other authors' findings (41).

Increased activity within the VIS may indicate an increased functional involvement of cortical structures in this area. This may be indicative of a compensatory mechanism associated with the physiological impairment of light signal perception and a decrease in the density of neurons in the retina and lateral geniculate bodies during ageing.

\section{Age-Related Increase in FC in Short-Range Connexions}

Moreover, we observed an age-related increase in RSFC in shortrange connexions such as between the nodes of left FPN lateral prefrontal cortex-DMN medial prefrontal cortex as well as the right DAN frontal eye field-DMN precuneus cortex. Similar results have also been reported in only one study (41). These findings seem to suggest that in older subjects RSFC increases between networks that show close anatomical proximity in the brain, while it decreases with age between networks that are located far away from each other in the brain. To the best of our knowledge, this is the second study indicating such a mechanism associated with the ageing process of the brain.

\section{Increased Functional Activity of the Cerebellum in Older Women}

Another interesting concept is related to cerebellar function which may lead to the hypothesis that the cerebellum could play a more important role in healthy ageing than previously supposed and can be assessed with the use of structural MRI. 
The cerebellum, thanks to numerous centripetal and centrifugal connexion pathways through the nuclei of the brainstem and the reticular system, plays a key role in automated regulatory processes that ensure maintaining balance, counteracting gravity, and coordinating deliberate voluntary movements and muscle tension (46). The cerebellum is also most likely involved in regulating cognitive and behavioural functions through its interactions with different cortical regions in different connectivity networks $(47,48)$. Several studies support the thesis that the cerebellum acts in cognition in the same fashion as cerebro-cerebellar connexions organised into long-range loops $(46,49)$. Previous studies on the structural characteristics of the human cerebellum were conducted in the context of a specific pathology or concerned a limited age range. In fact, research into life-long maturation and ageing of the cerebellum is rare, and most have considered the cerebellum as a whole without examining each lobule.

It seems that the increased activity of the cerebellum in older women may be related to the mechanism of recruiting more brain volume for cognitive purposes with age. The cerebellum most likely activates its long-range connexions with the rest of the cortical regions, including the prefrontal cortex, but it does not increase the speed of information transmission (46). Therefore, our data suggest that the cerebellum may be resistant to certain neurodegenerative mechanisms. To the best of our knowledge, our study is the first to present the importance of cerebellar network increase during healthy brain ageing in women.

\section{The Possible Mechanisms Associated With the Increased FC With Ageing}

We assume that the increased FC within some of the networks may reflect a compensatory mechanism; however, the increase could also be explained by neuronal excitotoxicity.

Neuronal excitotoxicity usually refers to the injury and death of neurons arising from prolonged exposure to glutamate which is the major excitatory neurotransmitter in human central nervous system. Excitotoxicity could be regarded also as a consequence of other cellular phenomena, such as mitochondrial dysfunction, physical neuronal damage, and oxidative stress (50). However, extracellular concentrations of glutamate in the human brain may also increase progressively with normal ageing (51).

Age-related changes may be caused by disruption of myelinated fibres that connect neurons in different cortical regions (52). In normal ageing, brain changes in the synaptic physiology of ageing neurons may contribute to altered connectivity (53). This process may contribute to compensatory increased neural activity in the older individuals, which could predispose the individuals to excitotoxicity and neurodegenerative pathology (54).

\section{Limitations}

There are some limitations to our study. First of all, we included a relatively small sample size, besides we evaluated RSFC changes only in women. Obviously, further studies are needed in order to compare age-related alterations in RSFC in men and women as well as to assess the ageing process involving the RSNs only in men. Nevertheless, the fact that our analysis was restricted only to women can be considered as a strength of this study. It has been suggested that the male and female brains age in a different way. According to this thesis, men and women should be evaluated separately in order to avoid any impact of sex on age-related RSFC changes. Although we started our research with female subjects, obviously we are going to study the intra- and inter-network RSFC differences between young and older male brains in the future as well.

Furthermore, our analysis was limited to eight main RSNs and main network nodes within each analysed network. However, we evaluated the cerebellar network that has often been omitted in other papers.

\section{Strengths of the Study}

On the other hand, it should be stressed that our study was performed using a modern 3-Tesla MR scanner equipped with gradients (45 $\mathrm{mT}$ and $200 \mathrm{~T} / \mathrm{m} / \mathrm{s}$ slew rate) with parallel and multislice acceleration and a 32-channel head coil resulting in a high signal-to-noise ratio achieved together with a relatively high spatial and temporal resolution of the acquired data.

In our study, time of scanning was optimised in order to achieve high intersession and intrasession reliability (55). Furthermore, the spatial resolution was a trade-off between SNR and temporal resolution (56).

Unfortunately, we cannot exactly quantify how short TR acquisition improved our results because that was not our null hypothesis. However, the effects of spatial resolution, increased acquisition time, and short TR on BOLD data have been widely discussed in the literature. Multislice rs-fMRI scan has improved the signal-to-noise ratio, while preserving the same level of the test-retest reliability compared to conventional EPI (57).

Most widely reported in the literature benefits of short TRs on stationary rs-fMRI metrics are minimal when compared with dynamic connectivity metrics. The analysis of dynamic connectivity should benefit more from shorter TRs than stationary metrics $(58,59)$.

We could only hypothesise that acquiring 800 timepoints and using multislice short TR acquisition improved our sensitivity and specificity similarly to the event-related fMRI studies available in the literature (60).

Moreover, we conducted the study using three different approaches to connectivity analysis. In our work, the main method of analysis was the ROI-ROI SPC (30). We also presented additional results obtained from different procedures such as FNC (27) and NBS (29). This approach allowed not only to reveal differences between the methods that we used but also to confirm our results within three different approaches used in the literature.

In summary, the results of our study seem to suggest that with older age, the brain appears to undergo a complicated reorganisation process associated with the integration and segregation of large-scale rs-networks. This assumption has also been proposed in other reports available in the literature $(41,61,62)$.

Overall, our findings suggest that RSFC changes in brain networks can serve as sensitive biomarkers for the age-related changes which are invisible in the structural MRI. Our results 
could support the idea that during ageing some inter-network connexions may function as a compensating mechanism to agerelated FC changes.

\section{CONCLUSIONS}

Our study seems to indicate a reorganisation mechanism of largescale functional brain networks during healthy female ageing, and it supports the evidence that apart from an overall reduction in RSFC between main rs-networks, ageing in women can lead to substantially increased FC between nodes of the default mode, the salience, the sensorimotor, the language, the fronto-parietal, the dorsal attention, and the VIS as well as the cerebellar anterior and cerebellar posterior networks.

It should be stressed that most of the reports on agerelated changes in the brain observed with the rs-fMRI studies concentrated on RSFC alterations within large-scale RSNs, except for the cerebellar network (CER). We assessed eight large-scale RSNs including the CER network. To the best of our knowledge, this study is the first to report the importance of cerebellar network increase during healthy ageing in women.

Moreover, little is known about age-related increase in RSFC within the VIS. We observed increased RSFC related to older age within the VIS in women and we also attempted to discuss the possible mechanisms responsible for these findings.

We believe that this project will contribute new knowledge to the comprehension of brain ageing while in the future our results could influence and improve the diagnosis and treatment of cognitive disorders in elderly people.

\section{DATA AVAILABILITY STATEMENT}

The original contributions presented in the study are included in the article/Supplementary Material, further inquiries can be directed to the corresponding author.

\section{REFERENCES}

1. Fischer KE, Riddle NC. Sex differences in aging: genomic instability. J Gerontol A Biol Sci Med Sci. (2018) 73:166-74. doi: 10.1093/gerona/glx105

2. Raz N, Gunning-Dixon F, Head D, Rodrigue KM, Williamson A, Acker JD. Aging, sexual dimorphism, and hemispheric asymmetry of the cerebral cortex: replicability of regional differences in volume. Neurobiol Aging. (2004) 25:377-96. doi: 10.1016/S0197-4580(03)00118-0

3. Goldstein JM, Seidman LJ, Horton NJ, Makris N, Kennedy DN, Caviness VS, et al. Normal sexual dimorphism of the adult human brain assessed by in vivo magnetic resonance imaging. Cereb Cortex. (2001) 11:4907. doi: $10.1093 /$ cercor/11.6.490

4. Goyal MS, Blazey TM, Su Y, Couture LE, Durbin TJ, Bateman RJ, et al. Persistent metabolic youth in the aging female brain. Proc Natl Acad Sci USA. (2019) 116:3251-5. doi: 10.1073/pnas.1815917116

5. Eugene AR, Masiak J. A pharmacodynamic modelling and simulation study identifying gender differences of daily olanzapine dose and dopamine D2-receptor occupancy. Nord J Psychiatry. (2017) 71:417-24. doi: 10.1080/08039488.2017.1314011

6. Zhang C, Dougherty CC, Baum SA, White T, Michael AM. Functional connectivity predicts gender: evidence for gender differences in resting brain connectivity. Hum Brain Mapp. (2018) 39:1765-76. doi: 10.1002/hbm.23950

\section{ETHICS STATEMENT}

The studies involving human participants were reviewed and approved by the Wroclaw Medical University Ethics Committee for conducting research involving humans no. of permission KB-57/2021. The patients/participants provided their written informed consent to participate in this study.

\section{AUTHOR CONTRIBUTIONS}

PP analyzed and interpreted rs-fMRI data, performed all the figures and tables, conducted literature search, and wrote the manuscript. MW-P contributed to the study design, wrote some parts of the manuscript, conducted literature search, and critically reviewed the paper. AZ contributed to the study design and critically reviewed the manuscript. MS contributed to the study design and critically reviewed the paper. JB, contributed to the study design, wrote some parts of the manuscript, supervised writing of the manuscript, critically reviewed the paper and supervised the research project. All authors contributed to the article and approved the submitted version.

\section{FUNDING}

This work was supported by Wroclaw Medical University Grant SUB.C270.21.020.

\section{SUPPLEMENTARY MATERIAL}

The Supplementary Material for this article can be found online at: https://www.frontiersin.org/articles/10.3389/fneur. 2021.645974/full\#supplementary-material

7. Weis S, Patil KR, Hoffstaedter F, Nostro A, Yeo T, Eickhoff SB. Sex classification by resting state brain connectivity. Cerebral Cortex. (2020) 30:824-35. doi: 10.1093/cercor/bhz129

8. Oksuzyan A, Juel K, Vaupel JW, Christensen K. Men: good health and highmortality. Sex differences in health and aging. Aging Clin Exp Res. (2008) 20:91-102. doi: 10.1007/BF03324754

9. Lv H, Wang Z, Tong E, Williams LM, Zaharchuk G, Zeineh M, et al. Resting-state functional MRI: everything that nonexperts have always wanted to know. AJNR Am J Neuroradiol. (2018) 39:1390-9. doi: 10.3174/ajnr. A5527

10. Ferreira LK, Busatto GF. Resting-state functional connectivity in normal brain aging. Neurosci Biobehav Rev. (2013) 37:384400. doi: 10.1016/j.neubiorev.2013.01.017

11. Dennis EL, Thompson, PM. Functional brain connectivity using fMRI in aging and Alzheimer's disease. Neuropsychol Rev. (2014) 24:4962. doi: 10.1007/s11065-014-9249-6

12. Ferreira LK, Regina AC, Kovacevic N, da Graça Morais Martin M, Santos PP, de Godoi Carneiro C, et al. Aging effects on whole brain functional connectivity in adults free of cognitive and psychiatric disorders. Cereb Cortex. (2016) 26: 3851-65. doi: 10.1093/cercor/bhv190

13. Jockwitz C, Caspers S, Lux S, Eickhoff SB, Jutten K, Lenzen S, et al. Influence of age and cognitive performance on resting-state brain networks 
of older adults in a population-based cohort. Cortex. (2017) 89:2844. doi: 10.1016/j.cortex.2017.01.008

14. Klaassens BL, van Gerven JMA, van der Grond J, de Vos F, Moller C, Rombouts S. Diminished posterior precuneus connectivity with the default mode network differentiates normal aging from Alzheimer's disease. Front Aging Neurosci. (2017) 9:97. doi: 10.3389/fnagi.2017.00097

15. Betzel R, Byrge L, He Y, Goni J, Zuo XN, Sporns O. Changes in structural and functional connectivity among resting-state networks across the human lifespan. Neuroimage. (2014) 102 (Pt 2):345-57. doi: 10.1016/j.neuroimage.2014.07.067

16. Chan MY, Park DC, Savalia NK, Petersen SE, Wig GS. Decreased segregation of brain systems across the healthy adult lifespan. Proc Natl Acad Sci USA. (2014) 111, E4997-5006. doi: 10.1073/pnas.1415122111

17. Geerligs L, Renken RJ, Saliasi E, Maurits NM, Lorist MM. A brain-wide study of age-related changes in functional connectivity. Cereb Cortex. (2015) 25:1987-99. doi: 10.1093/cercor/bhu012

18. Grady C, Sarraf S, Saverino C, Campbell K. Age differences in the functional interactions among the default, frontoparietal control, and dorsal attention networks. Neurobiol Aging. (2016) 41:159-72. doi: 10.1016/j.neurobiolaging.2016.02.020

19. Friston KJ, Ashburner J, Kiebel SJ, Nichols TE, Penny WD, (Eds). Statistical Parametric Mapping: The Analysis of Functional Brain Images. Cambridge, MA: Academic Press (2007).

20. Andersson JLR, Hutton C, Ashburner J, Turner R, Friston K. Modelling geometric deformations in EPI time series. NeuroImage. (2001) 13:90919. doi: $10.1006 /$ nimg. 2001.0746

21. Henson RNA, Buechel C, Josephs O, Friston KJ. The slicetiming problem in event-related fMRI. NeuroImage. (1999) 9:125.

22. Ashburner J, Friston KJ. Unified segmentation. NeuroImage. (2005) 26:83951. doi: 10.1016/j.neuroimage.2005.02.018

23. Behzadi Y, Restom K, Liau J, Liu TT. A component based noise correction method (CompCor) for BOLD and perfusion based fMRI. NeuroImage. (2007) 37:90-101. doi: 10.1016/j.neuroimage.2007.04.042

24. Hallquist M, Hwang K, Luna B. The nuisance of nuisance regression: spectral misspecification in a common approach to resting-state fMRI preprocessing reintroduces noise and obscures functional connectivity. NeuroImage. (2013) 82:208-25. doi: 10.1016/j.neuroimage.2013.05.116

25. Nieto-Castanon A. Handbook of Functional Connectivity Magnetic Resonance Imaging Methods in CONN. Boston, MA: Hilbert-Press (2020).

26. Stanley M, Moussa M, Paolini B, Lyday R, Burdette J, Laurienti P. Defining nodes in complex brain networks. Front Comput Neurosci. (2013) 7:169. doi: $10.3389 /$ fncom.2013.00169

27. Jafri MJ, Pearlson GD, Stevens M, Calhoun VD. A method for functional network connectivity among spatially independent resting-state components in schizophrenia. NeuroImage. (2008) 39:1666-81. doi: 10.1016/j.neuroimage.2007.11.001

28. Benjamini Y, Hochberg Y. Controlling the false discovery rate: a practical and powerful approach to multiple testing. J R Statist Soc B. (1995) 57:289300. doi: 10.1111/j.2517-6161.1995.tb02031.x

29. Zalesky A, Cocchi L, Fornito A, Murray MM, Bullmore E. Connectivity differences in brain networks. NeuroImage. (2012) 60:1055-62. doi: 10.1016/j.neuroimage.2012.01.068

30. Zalesky A, Fornito A, Bullmore ET. Network-based statistic: identifying differences in brain networks. NeuroImage. (2010) 53:1197-207. doi: 10.1016/j.neuroimage.2010.06.041

31. Achard S, Bullmore E. Efficiency and cost of economical brain functional networks. PLoS Comput Biol. (2007) 3:e17. doi: 10.1371/journal.pcbi.0030017

32. Batouli AH, Boroomand A, Fakhri M, Sikaroodi H, Oghabian MA, Firouznia $\mathrm{K}$. The effect of aging on resting-state brain function: an fMRI study. Iran $J$ Radiol. (2009) 6:153-8.

33. Bluhm RL, Osuch EA, Lanius RA, Boksman K, Neufeld RW, Theberge J, et al. Default mode network connectivity: effects of age, sex, and analytic approach. Neuroreport. (2008) 19:887-91. doi: 10.1097/WNR.0b013e328300ebbf

34. Damoiseaux JS, Beckmann CF, Arigita EJ, Barkhof F, Scheltens P, Stam CJ, et al. Reduced resting-state brain activity in the default network in normal aging. Cerebral Cortex. (2008) 18:1856-64. doi: 10.1093/cercor/bhm207

35. Esposito F, Aragri A, Pesaresi I, Cirillo S, Tedeschi G, Marciano E, et al. Independent component model of the default-mode brain function: combining individual-level and population-level analyses in resting-state fMRI. Magn Reson Imaging. (2008) 26:905-13. doi: 10.1016/j.mri.2008.01.045

36. Koch W, Teipel S, Mueller S, Buerger K, Bokde AL, Hampel H, et al. Effects of aging on default mode network activity in resting state fMRI: does the method of analysis matter? NeuroImage. (2010) 51:2807. doi: 10.1016/j.neuroimage.2009.12.008

37. Tomasi D, Volkow ND. Aging and functional brain networks. Mol Psychiatry. (2012) 17:549-58. doi: 10.1038/mp.2011.81

38. Wang L, Laviolette P, O'Keefe K, Putcha D, Bakkour A, Van Dijk KR, et al. Intrinsic connectivity between the hippocampus and posteromedial cortex predicts memory performance in cognitively intact older individuals. NeuroImage. (2010) 51:910-7. doi: 10.1016/j.neuroimage.2010.02.046

39. Jones DT, Machulda MM, Vemuri P, McDade EM, Zeng G, Senjem ML, et al. Age-related changes in the default mode network are more advanced in Alzheimer disease. Neurology. (2011) 77:1524-31. doi: 10.1212/WNL.0b013e318233b33d

40. Westlye ET, Lundervold A, Rootwelt H, Lundervold AJ, Westlye LT. Increased hippocampal default mode synchronisation during rest in middle-aged and elderly APOE epsilon4 carriers: relationships with memory performance. $J$ Neurosci. (2011) 31:7775-83. doi: 10.1523/JNEUROSCI.1230-11.2011

41. Zonneveld HI, Pruim RHR, Bos D, Vrooman HA, Muetzel RL, Hofman A, et al. Patterns of functional connectivity in an aging population: the Rotterdam study. NeuroImage. (2019) 189:432-44. doi: 10.1016/j.neuroimage.2019.01.041

42. Ritchie SJ, Cox SR, Shen X, Lombardo MV, Reus LM, Alloza C, et al. Sex differences in the adult human brain: evidence from 5,216 UK biobank participants. Cerebral Cortex. (2018) 28:2959-75. doi: 10.1093/cercor/bhy109

43. Casco C, Robol V, Barollo M, Cansino S. Effects of aging on visual contour integration and segmentation. Invest Ophthalmol Vis Sci. (2011) 52:395561. doi: 10.1167/iovs.10-5439

44. Bagarinao E, Watanabe $\mathrm{H}$, Maesawa, S. Reorganization of brain networks and its association with general cognitive performance over the adult lifespan. Sci Rep. (2019) 9 11352. doi: 10.1038/s41598-019-47922-x

45. Park DC, Reuter-Lorenz P. The adaptive brain: aging and neurocognitive scaffolding. Annu Rev Psychol. (2009) 60:17396. doi: 10.1146/annurev.psych.59.103006.093656

46. Liang KJ, Carlson ES. Resistance, vulnerability and resilience: a review of the cognitive cerebellum in aging and neurodegenerative diseases. Neurobiol Learn Mem. (2020) 170:106981. doi: 10.1016/j.nlm.2019. 01.004

47. Houck BD, Person AL. Cerebellar premotor output neurons collateralize to innervate the cerebellar cortex. J Comp Neurol. (2015) 523:225471. doi: $10.1002 /$ cne. 23787

48. Buckner RL. The cerebellum and cognitive function: 25 years of insight from anatomy and neuroimaging. Neuron. (2013) 80:807-15. doi: 10.1016/j.neuron.2013.10.044

49. Watson TC. Back to front: cerebellar connections and interactions with the prefrontal cortex. Front Syst Neurosci. (2014) 8:4. doi: 10.3389/fnsys.2014.00004

50. Armada-Moreira A, Gomes JI, Campos Pina C, Savchak OK, GonçalvesRibeiro J, Rei N, et al. Going the extra (synaptic) mile: excitotoxicity as the road toward neurodegenerative diseases. Front Cell Neurosci. (2020) 14:90. doi: 10.3389/fncel.2020.00090

51. Castelli V, Benedetti E, Antonosante A, Catanesi M, Pitari G, Ippoliti R, et al. Neuronal cells rearrangement during aging and neurodegenerative disease: metabolism, oxidative stress and organelles dynamic. Front Mol Neurosci. (2019) 12:132. doi: 10.3389/fnmol.2019.00132

52. Andrews-Hanna JR, Snyder AZ, Vincent JL, Lustig C, Head D, Raichle ME, et al. Disruption of large-scale brain systems in advanced aging. Neuron. (2007) 56:924-35. doi: 10.1016/j.neuron.2007.10.038

53. Yankner BA, Lu T, Loerch P. The aging brain. Annu Rev Pathol. (2008) 3:41-66. doi: 10.1146/annurev.pathmechdis.2.010506.092044

54. Bishop NA, Lu T, Yankner BA. Neural mechanisms of ageing and cognitive decline. Nature. (2010) 464:529-35. doi: 10.1038/nature08983

55. Birn RM, Molloy EK, Patriat R, Parker T, Meier TB, Kirk GR, et al. The effect of scan length on the reliability of resting-state fMRI connectivity estimates. NeuroImage. (2013) 83:550-8. doi: 10.1016/j.neuroimage.2013. 05.099 
56. Molloy EK, Meyerand ME, Birn RM. The influence of spatial resolution and smoothing on the detectability of resting-state and task fMRI. NeuroImage. (2014) 86:221-30. doi: 10.1016/j.neuroimage.2013.09.001

57. Jahanian $\mathrm{H}$, Holdsworth $\mathrm{S}$, Christen $\mathrm{T}$, Wu H, Zhu K, Kerr AB, et al. Advantages of short repetition time resting-state functional MRI enabled by simultaneous multi-slice imaging. J Neurosci Methods. (2019) 311:12232. doi: 10.1016/j.jneumeth.2018.09.033

58. Golestani AM, Kwinta JB, Khatamian YB, Chen JJ. The effect of low-frequency physiological correction on the reproducibility and specificity of restingstate fMRI metrics: functional connectivity, ALFF, and ReHo. Front Neurosci. (2017) 11:546. doi: 10.3389/fnins.2017.00546

59. Huotari N, Raitamaa L, Helakari H, Kananen J, Raatikainen V, Rasila A, et al. Sampling rate effects on resting state fMRI metrics. Front Neurosci. (2019) 13:279. doi: 10.3389/fnins.2019.00279

60. McDowell AR, Carmichael DW. Optimal repetition time reduction for single subject event-related functional magnetic resonance imaging. Magn Reson Med. (2019) 81:1890-7. doi: 10.1002/mrm.27498
61. Sala-Llonch R, Bartres-Faz D, Junque C. Reorganization of brain networks in aging: a review of functional connectivity studies. Front Psychol. (2015) 6:663. doi: 10.3389/fpsyg.2015.00663

62. Sun J, Tong S, Yang GY. Reorganization of brain networks in aging and age-related diseases. Aging Dis. (2012) 3:181-93.

Conflict of Interest: The authors declare that the research was conducted in the absence of any commercial or financial relationships that could be construed as a potential conflict of interest.

Copyright (c) 2021 Podgórski, Waliszewska-Prosół, Zimny, Sąsiadek and Bladowska. This is an open-access article distributed under the terms of the Creative Commons Attribution License (CC BY). The use, distribution or reproduction in other forums is permitted, provided the original author(s) and the copyright owner(s) are credited and that the original publication in this journal is cited, in accordance with accepted academic practice. No use, distribution or reproduction is permitted which does not comply with these terms. 